SaberEs. Vol. 11 Núm. 1 (2019).65-80. Sección Artículos

\title{
ANÁLISIS PRELIMINAR DE LA CADENA DE VALOR DE LA ROSA MOSQUETA EN BARILOCHE Y ZONA DE INFLUENCIA, ARGENTINA ${ }^{1}$
}

\author{
Juan Martín Quiroga*
}

Resumen. Este trabajo analiza la actividad económica desarrollada a partir de la Rosa Mosqueta en la región noroeste de la Patagonia Argentina, buscando comprender cada una de las actividades involucradas. Para ello se recurre al enfoque de las Cadenas Globales de Valor, marco que se profundizó con un análisis de las tecnológicas involucradas en cada una de las etapas. La investigación se basó en la utilización de fuentes secundarias y primarias, particularmente la entrevista a un empresario del sector. En las conclusiones se destaca la existencia de un cuello de botella en la recolección del fruto y se argumenta que es el resultado de la asimetría entre recolectores y acopiadores. Por ello se propone la asociatividad de los recolectores a fin de mejorar su poder de negociación y se discute el posible rol que el desarrollo de tecnologías de cosecha ad-hoc podría tener para este fin. Se espera que este artículo constituya un insumo para decisiones de actores sociales y empresarios del sector, así como también para el desarrollo de políticas públicas por parte de funcionarios y agentes gubernamentales.

Palabras Clave: Cadenas Globales de Valor; Economías Regionales; Tecnologías sociales.

\footnotetext{
* Universidad Nacional de Río Negro (UNRN), Sede Andina, Argentina.

Contacto: jquiroga@unrn.edu.ar

1 La investigación que dio lugar a este trabajo fue realizada en el marco de la participación del autor como consultor en el Programa Nacional de Apoyo al Relevamiento de Demandas Tecnológicas (Programa PAR), financiado por el Ministerio de Ciencia, Tecnología e Innovación Productiva de la Nación, Argentina. Asimismo se desea agradecer tanto los comentarios como la minuciosa revisión realizada por el/la Evaluador/a 1.
} 


\title{
A PRELIMINARY VALUE CHAIN ANALYSIS OF THE ROSE HIP IN BARILOCHE AND INFLUENCE AREA, ARGENTINA
}

\begin{abstract}
This paper analyzes the economic activity related to the Rose Hip in the north-western region of the Argentine Patagonia, with the aim of understanding every activity involved. For this purpose, the Global Value Chains approach is used, deepened with an analysis of the technology involved in each of these stages. The conclusions highlight the existence of a bottleneck in the harvesting of Rose Hip, and it is argued that it is the result of the asymmetry between harvesters and collectors. Therefore, the associativity of harvesters is proposed in order to improve their bargaining power, and the possible role that the development of ad-hoc harvest technologies could have for this purpose is discussed. It is expected that this article constitutes an input for decision making of social actors and businessman that belongs to this sector, as well as for the development of public policies by government officials and agents.
\end{abstract}

Keywords: Global Value Chains; Regional Economies; Social Technologies.

Original recibido el 26/02/2018

Aceptado para su publicación el 11/07/2018 


\section{Introducción}

La Rosa Mosqueta (Rosa eglantería, y también Rosa canina) es una planta originaria de Europa que en la región cordillerana de la Patagonia argentina, y algunas zonas de Mendoza, constituye una especie invasora. Esta planta es conocida desde la antigüedad por las propiedades nutricionales y medicinales de sus frutos, entre las que resalta el alto contenido de vitamina $C$. En la región Oeste de la Provincia de Río Negro, en Argentina, se sitúa la ciudad de San Carlos de Bariloche y su zona de influencia ${ }^{2}$ y es donde se da el mayor aprovechamiento agroindustrial de esta planta en la Argentina. Sin embargo, y en forma contraria a lo que sucede en otros países (como Chile, España o Inglaterra) donde es cultivada, su explotación en nuestro país se realiza a partir de la recolección de frutos silvestres de las plantas que crecen invadiendo el sotobosque nativo o las praderas esteparias y precordilleranas.

Argentina se fue constituyendo paulatinamente en un exportador de materia prima y productos elaborados a partir de la Rosa Mosqueta, pese a la falta de cultivos sistematizados. Los mercados de destino incluyen países como Alemania, Chile, China y Corea del Sur, en los cuales son utilizados en la industria alimenticia, cosmética y farmacéutica, existiendo, además, perspectivas de utilización para fines nutracéuticos (Entrevista a empresario del sector, 2016).

En la actualidad no se cuenta con estadísticas oficiales respecto a los volúmenes cosechados que permitan dimensionar adecuadamente la actividad de recolección, principalmente debido al carácter informal de esta etapa de la cadena de valor. Sin embargo, estimaciones del año 2005 realizadas por el Instituto Nacional de Tecnología Agropecuaria (INTA) indicaban que en las regiones aledañas a las ciudades de Bariloche, El Bolsón y Esquel, se cosechaban aproximadamente unas $1.400 \mathrm{t}$ anuales de frutos. Estas mismas estadísticas indican que existiría la posibilidad de ampliar dicha cosecha a unas $20.000 \mathrm{t}$ (INTA - SIPAN, 2005), extendiéndose las poblaciones de esta planta entre los $36^{\circ}$ y $44^{\circ}$ de Latitud Sur (Damascos, 2008).

Respecto a la utilización en agroindustrias vinculadas a la Rosa Mosqueta, en los últimos años se ha experimentado un incremento de la demanda local, tanto de frutos como de productos derivados. De acuerdo a datos ofrecidos por un empresario del sector, los pedidos recibidos en 2015 llegan a más de 300 t de semillas anuales. Cabe mencionarse que se necesitan grandes volúmenes de frutos y semillas para obtener productos como dulces y aceites: $1 \mathrm{~kg}$ de semilla se obtiene de aproximadamente $4,5 \mathrm{~kg}$ de frutos frescos, por lo que la provisión de las $300 \mathrm{t}$ de semillas implicaría la cosecha a $1.350 \mathrm{t}$ de frutos. Si a ello se suman los pedidos por aceite y otros productos procesados, se puede obtener una estimación aproximada de la demanda insatisfecha y del potencial de crecimiento del sector: 1 I de aceite se obtiene del prensado de $33 \mathrm{~kg}$ de semillas lo cual implica cosechar $150 \mathrm{~kg}$ de frutos frescos. El incremento en la demanda de la Rosa Mosqueta y sus productos derivados, pone en evidencia que actualmente la Cadena de Valor de la Rosa Mosqueta (CVRM) muestra un cuello de botella ocasionado por el bajo volumen de recolección.

2 Región que abarca la zona cordillerana del sur de la provincia de Neuquén y noroeste de la de Chubut. 


\section{Marco teórico}

El concepto de "cadena de valor", acuñado por Michael Porter (1980; 1985), se fue difundiendo desde su introducción en la década de 1980. Una explicación acotada del mismo consiste en que este enfoque permite analizar los procesos productivos y comerciales de las empresas a partir de una sucesión de eslabones, es decir las etapas u operaciones necesarias para transformar materias primas e insumos en productos y servicios (Kaplinsky y Morris, 2000). Por ello, en dicho análisis es necesario tomar en consideración aquellas actividades tanto previas como posteriores a la manufactura. Al análisis de ese grupo de actividades centrales, se le agrega el de otro conjunto de actividades de soporte o secundarias.

Con el tiempo fueron surgiendo otros enfoques, en los que se desarrolló y complejizó el concepto de "cadena" (de valor), como se aprecia en los estudios de "cadenas globales de commodities" de la década de 1990, tales como los trabajos de Gereffi $(1994,1995,1999)$ y en los desarrollos posteriores de "cadenas globales de valor" (Gereffi et al., 2001; 2005). Ya en sus primeros trabajos, este autor destaca la existencia de cuatro dimensiones centrales de análisis para el estudio de las cadenas globales de valor: i) la estructura de insumo-producto (es decir la concatenación de actividades productivas a través de la cual se va agregando y distribuyendo el valor, por medio de la fabricación de productos y la prestación de servicios); ii) la estructura de coordinación y poder (governance); iii) la territorialidad, o alcance geográfico, de la cadena; y iv) el contexto institucional.

Además de la necesidad de tener en cuenta los cuatro aspectos mencionados anteriormente, también cabe aclararse que si bien la representación gráfica de la cadena de valor puede llevar a una interpretación lineal de su funcionamiento, en la práctica las dinámicas vinculadas suelen no tener un carácter lineal. La producción per se comprende sólo una parte de todos los eslabones de la cadena y dentro de cada eslabón se puede encontrar además un conjunto amplio y diverso de actividades. Por ello las vinculaciones entre eslabones no se dan únicamente en un sentido, existiendo feedbacks entre diversos eslabones, por ejemplo, entre el diseño del producto y las actividades de marketing y fabricación. Asimismo, las cadenas de valor no están aisladas del resto de la actividad económica, y por lo tanto cabe esperarse que los diversos eslabonamientos pertenezcan a más de una cadena de valor, generándose un entrelazamiento entre las mismas.

Los enfoques recién mencionados se han desarrollado sobre la base de la internacionalización, reestructuración y relocalización a nivel global de distintos eslabones de los circuitos productivos y comerciales. Sin embargo, como indican Civitaresi, Niembro y Sarmiento (2015), los aportes metodológicos también pueden aprovecharse flexibilizando la aplicación a "cadenas/redes domésticas", haciendo una interpretación amplia del comercio y las "exportaciones" de una región particular, para analizar la interacción entre la dimensión local y la escala extraregional (factores nacionales y/o globales).

Por último, no hay que perder de vista que los diferentes componentes de la cadena de valor se encuentran insertos y arraigados en locaciones específicas con contextos sociopolíticos, institucionales y culturales particulares y que estos factores influyen 
sobre la propia naturaleza y dinámica de la cadena. La relevancia de la estructura de instituciones formales (leyes, normas, regulaciones) e informales (convenciones, valores, costumbres) como parte de una noción más abarcativa, sistémica y dinámica de las cadenas (globales) de valor puede rastrearse en trabajos como el de Gereffi et al. (2005).

\section{Rosa Mosqueta: una planta, dos percepciones}

En las zonas aledañas a la ciudad de San Carlos de Bariloche, la Rosa Mosqueta tiene una valoración ambivalente. Por un lado, existe evidencia científica de sus excelentes propiedades, tal como detallan Espinoza et al. (2016), lo cual da lugar a la existencia de una cadena de valor que genera trabajo local y ventas extraregionales y exportación de materias primas y productos derivados. Por otro lado, esta misma planta encuentra un amplio rechazo por sectores de la sociedad que la asocian únicamente con su carácter de maleza invasora (Damascos, 2008). Dentro de este sector es particularmente relevante la posición respecto al "combate" a la maleza existente entre los productores agropecuarios. Este grupo social históricamente se ha opuesto al ensayo de cultivos sistematizados de la misma al considerar que aumentaría aún más la propagación de esta especie invasora, que paulatinamente inhabilita áreas de pastura para el ganado.

Estas posiciones antagónicas son reflejadas también en la escasa literatura científica que ha abordado la temática, en la cual también es común encontrar estas tensiones. Por un lado, existen estudios que analizan la cuestión haciendo frente a los aspectos negativos de la expansión de esta especie exótica. En ellos se plantea la problemática derivada del avance paulatino en campos en los que se realizan explotaciones agropecuarias y, por ello, los mismos se enfocan en experimentar diversos tratamientos de control eficientes contra la Rosa Mosqueta tales como la extracción mecánica (INTA AER EI Bolsón, 2013) o el uso de agroquímicos como el glifosato (Lloyd, 2014). La otra postura, respecto a los potenciales aprovechamientos socioeconómicos de la Rosa Mosqueta, apuntan a la creación de circuitos agroindustriales (Madariaga, 2007; Ohaco, Mabellini, y Márquez, 2011) o proponen desarrollos tecnológicos vinculados a ella (Cattan Lavin, 2007; PDTT 2016a, 2016b, 2016c y 2017).

Los argumentos que sustentan el interés socioeconómico de la Rosa Mosqueta están basados en el valor económico de sus productos derivados, que son ricos en Vitaminas A, C (veinte veces superior al de la naranja) y $\mathrm{F}$; minerales como Fósforo, Potasio, Calcio, Magnesio, Sodio, Hierro, Cobre, Manganeso, Zinc; ácidos tales como oléico, linoléico y linolénico, entre otros; y pigmentos como carotenoides, riboflaminas y pectinas (Espinoza et al., 2016; Damascos, 2008). Asimismo se ha demostrado que posee efectos antioxidantes, antiinflamatorios, antidiabéticos y como agente antibacteriano (para un resumen de las investigaciones respecto a las propiedades ver Patel, 2013). También se ha investigado su uso como complemento quimioterápico, para tratamientos dermatológicos (por sus propiedades cicatrizantes y regenerantes de tejido) y en medicina natural, donde son conocidos sus beneficios para el tratamiento de la gripe, la depresión, la disolución de cálculos y limpieza de riñones y vejiga (Espinoza et al., 2016). 


\section{La cadena de valor de la rosa mosqueta}

\subsection{La estructura de insumo-producto}

\subsubsection{Recolección}

La cadena de valor de la Rosa Mosqueta se inicia con la cosecha manual y atomizada de frutos por parte de grupos de recolectores, generalmente pertenecientes a sectores vulnerables de la población que encuentran en su venta a acopiadores y elaboradores de productos derivados ${ }^{3}$ una fuente de ingresos adicionales. Estas actividades se realizan durante el período otoñal (marzo a junio aproximadamente). La metodología generalmente utilizada por los recolectores más organizados, consiste en adentrarse en campos y tierras fiscales a la vera de las rutas de la región, cosechar los frutos en bolsas (tipo de arpillera) y arrastrarlas hasta las rutas donde son cargadas en algún medio de transporte.

Esta actividad en la actualidad constituye el principal cuello de botella en la CVRM $y$, desde la perspectiva de los compradores del fruto, conlleva la existencia de una alta incertidumbre respecto a los volúmenes que efectivamente se cosecharán cada año. A su vez, si se analiza desde la perspectiva de los recolectores, que poseen un bajo poder de negociación y constituyen una pléyade de actores individuales frente a pocos acopiadores y procesadores, se genera también incertidumbre respecto al precio que efectivamente cobrarán luego de la recolección. El resultado de esta incertidumbre, es que se tienen pocos incentivos para incrementar los esfuerzos de recolección o aumentar la cantidad de recolectores.

\subsubsection{Acopio, acondicionamiento y procesamiento 4}

Los frutos recolectados son comprados por acopiadores. El precio pagado por estos varía principalmente por la calidad de lo cosechado: puesto que para la elaboración de subproductos se necesita solo el fruto, la presencia de hojas y cabos de los frutos provoca la disminución del precio de compra, dado que implican un procesamiento adicional a fin de retirarlos. Por la información que pudo relevarse, la separación de hojas y cabos se realiza en forma manual.

El principal acopiador y acondicionador del fruto de Rosa Mosqueta es un secadero localizado en la ciudad de Lago Puelo, Provincia de Chubut, Argentina. Por el gran volumen que maneja cada año, el precio regional de los frutos suele ser fijado por esta empresa. En la campaña 2016, el kg limpio (deshojado y sin cabos) de Rosa Mosqueta se pagó \$6.

Los frutos de Rosa Mosqueta son aprovechados en su totalidad para la elaboración de productos alimenticios, nutracéuticos y cosméticos. Dependiendo del tipo de productos para el que se vaya a destinar, es posible realizar un procesamiento con el fruto fresco o bien con el fruto previamente secado. En el primer caso, la cascarilla separada del fruto se utiliza para elaboración de pulpa de frutas y las semillas para su prensado ${ }^{5}$. En el segundo caso, es necesario proceder a secar el fruto, separar la

3 Véase la Tabla 1, para un detalle de productos derivados de la Rosa Mosqueta.

4 Estas tres actividades han sido unificadas en un solo acápite puesto que son realizadas por los mismos actores.

5 Esto se profundiza en el apartado siguiente. 
cascarilla de las semillas y realizar su prensado.

Estas últimas actividades suelen realizarse con instalaciones y maquinarias desarrollas para tal fin. En el caso del secado, suele recurrirse a hornos de microondas industriales o al secado por convección de aire caliente, lo cual se logra gracias a la utilización de mecheros de gas. Respecto al prensado, el mismo puede ser en frío, lo cual permite obtener un aceite de mayor calidad, o por medio de reactivos químicos, en los cuales el producto obtenido es de menor calidad y no puede ser certificado de orgánico. Un procesamiento adicional que ha surgido recientemente es la molienda de las briquetas, subproducto del prensado para la obtención de aceite.

\subsubsection{Elaboración de productos derivados}

La Rosa Mosqueta se utiliza para la producción de una amplia gama de productos. EI fruto se utiliza para la elaboración de licores y vinos, la pulpa y la cascarilla (cáscara deshidratada) son utilizadas para productos alimenticios como dulces, infusiones y suplementos proteicos. Por su parte, las semillas son utilizadas para elaborar aceite, un producto muy valorado en la industria cosmética y farmacéutica por sus propiedades antioxidantes (Barros Biscari, 2010; Damascos, 2008; Espinoza et al., 2016; Madariaga, 2007). Asimismo, históricamente las briquetas, subproducto del prensado de las semillas, se han utilizado como combustible, particularmente en Chile (Barros Biscari, 2010). También han sido ensayadas para la elaboración de biogás (Espinoza et al., 2016), son utilizadas como alimento para cerdos, chinchillas y pollos (Damascos, 2008) y, desde 2015, una empresa de Bariloche asistida por el Instituto Nacional de Tecnología Industrial (INTI) ha comenzado a experimentar con harina de Rosa Mosqueta. Este es un producto apto para celíacos y con potenciales propiedades nutracéuticas, el cual es elaborado en base a la molienda de las briquetas con resultados alentadores (Entrevista a empresario del sector, 2016).

En tanto que los productos alimenticios y los aceites esenciales suelen ser elaborados en Bariloche y la zona de influencia, los productos cosméticos son elaborados por laboratorios ubicados en la Ciudad Autónoma de Buenos Aires y luego, una parte de ellos, son reenviados a Bariloche para su comercialización. Esto es así por el hecho de que las exigencias para la certificación de laboratorios por parte de la Administración Nacional de Medicamentos, Alimentos y Tecnología Médica (ANMAT) han sido un factor limitante para el asentamiento de laboratorios en la ciudad de Bariloche (Entrevista a empresario del sector, 2016).

\subsubsection{Comercialización}

Existen diversos mercados para los productos en base a Rosa Mosqueta. Una primera distinción lleva a considerar el mercado mayorista y el minorista. En el caso del mercado mayorista, en los últimos años se han registrado exportaciones de semillas, cascarilla y aceite a Chile, más allá de las ventas que realizan los acopiadores a los productores de alimentos y cosméticos. Asimismo, un empresario local ha mencionado que recibe ofertas de países como China y Corea del Sur para comprar este tipo de productos con bajo valor agregado. En esta categoría también puede situarse la comercialización de las briquetas, subproducto de la elaboración de aceites por prensado, antes mencionada. 
Los productos comercializados con mayor agregado de valor, vinculados a canales minoristas, pueden agruparse en dos grandes categorías: productos alimenticios, donde se incluye la venta de cascarilla y pulpas a otros entramados productivos (particularmente para la elaboración de tés regionales y productos alimenticios como dulces, chocolates, helados y alfajores) y productos cosméticos. Este tipo de producto es vendido en locales comerciales de las localidades de Bariloche y zona de influencia, y en algunos comercios de grandes ciudades, como Buenos Aires, Córdoba o Rosario. También cabe mencionar que el canal minorista ha aprovechado las ventajas que ofrece la venta de productos on-line, siendo posible realizar compras por internet.

\section{Tabla 1. Productos elaborados en base a Rosa Mosqueta}

\begin{tabular}{cc} 
Productos Alimenticios & Productos Cosméticos \\
\hline Licor & Aceite \\
Vino & Cremas \\
Pulpa & Bálsamo labial \\
Dulces & \\
Sopas & \\
Té & \\
Suplementos protéicos & \\
Harina &
\end{tabular}

Fuente: elaboración propia en base a Madariaga (2007), Damascos (2008), Barros Biscari (2010) y Espinoza et al. (2016).

\subsection{El factor tecnológico como componente de la CVRM}

En términos generales puede afirmarse que, por el carácter marginal que tiene la actividad para la economía regional sumado a la informalidad que caracteriza la recolección del fruto, el tipo de tecnologías utilizadas en esta cadena de valor es rudimentario, aunque adecuadas para explotaciones de tipo artesanales o de baja escala.

Respecto al eslabón de la recolección, la cosecha manual se realiza con herramientas en extremo sencillas desarrolladas en forma ad-hoc por los mismos cosechadores, tales como rastrillos a los que se les acorta el mango. En este sentido, en el marco del Programa PAR, del Ministerio de Ciencia, Tecnología e Innovación Productiva de la Nación, se han identificado Demandas de Innovación (DDIs) tendientes a aumentar tanto la seguridad personal como la productividad de los recolectores de frutos, proponiéndose el desarrollo de herramientas tanto manuales como mecánicas portátiles específicas para mejorar la productividad de la cosecha (PDTT, 2016a y 2016b).

Al interior de las actividades de acopio, acondicionamiento y procesamiento existen diferencias en el uso de tecnologías. Tanto el acopio como el acondicionamiento son actividades principalmente manuales, exceptuando el secado que se realiza por medio de los ya mencionados secaderos a gas y a microondas, sin superar los $70^{\circ} \mathrm{C}$ de temperatura. Cabe señalar que, puesto que la pulpa posee una cutícula 
muy dura y un importante recubrimiento céreo, la salida de vapor de agua durante el secado es muy lenta. En este sentido la necesidad de hacer más eficiente este proceso se ha plasmado en una DDI relevada en el marco del Programa PAR (PDTT, 2017), para el cual existe el antecedente del estudio de Ohaco, Mabellini, y Márquez (2011) en el cual demuestran que la realización de cortes y perforaciones de los frutos antes del secado, permite mejorar un $60 \%$ su tiempo, sin implicar el mismo una modificación de las características organolépticas del fruto y aumentando en un $140 \%$ la productividad de los equipos utilizados. Al momento de esta investigación, no se ha identificado que se haya implementado ninguna innovación tendiente a aumentar la eficiencia en el secado de los frutos.

El procesamiento es realizado por medio de diversas maquinas e instalaciones. El primer procedimiento es el quebrado, que se realiza en molinos especiales tratando de romper la fruta en trozos grandes, que permitan la separación de semillas y otras impurezas restantes. A continuación del quebrado, el resultado de dicha operación se pasa a una zaranda vibratoria en la que se separa la cascarilla más grande. El resto ingresa a otros sistemas de venteo de aire donde se procede a separar cascarilla y semillas gracias a su diversa densidad (Cameroni, 2013).

El prensado de semillas es la operación por la cual se obtienen, además del aceite, las briquetas que, gracias a una posterior molienda, permiten obtener harina de Rosa Mosqueta. Por último, cabe mencionar que la pulpa que se obtiene del procesamiento de la cascarilla de frutos que no han sido secados suele congelarse para su conservación.

\subsection{La estructura de coordinación y poder}

Este análisis preliminar de la estructura de coordinación y poder (governance) en la CVRM de Bariloche, ha permitido identificar que la actual estructura de coordinación y poder imperante entre los eslabonamientos de recolección y acopio constituye un importante cuello de botella para el crecimiento de esta cadena de valor. Es por ello que este apartado se focaliza en las relaciones asimétricas entre los recolectores y acopiadores, puesto que se ha identificado que en el vínculo entre estos dos eslabones es donde la cadena de valor no logra superar un factor crítico limitante que es la disponibilidad de materia prima para su procesamiento. Vinculado a ello, otro aspecto a considerar (y a su vez derivado del carácter informal de la recolección) es la incertidumbre respecto a la cantidad que se cosechará cada año.

Las asimetrías existentes entre unos recolectores atomizados y poco organizados, respecto a los acopiadores, concentrados en unos pocos actores, dan como resultado un escaso poder de negociación de los primeros, quienes no obtienen un precio atractivo para el fruto de su trabajo, realizado en la época de lluvias, con bajas temperaturas, y teniendo que lidiar con una planta que tiene muchas espinas. Esta situación conlleva a que no existan muchos incentivos para que se incremente el volumen de recolección de Rosa Mosqueta, reproduciéndose en el tiempo el carácter de actividad vinculada a sectores sociales marginales, que recurren a esta actividad informal como complemento de otras actividades económicas de subsistencia. Esta situación es aprovechada por los acopiadores, que no tienen mayores problemas en establecer un precio bajo para una materia prima utilizada para elaborar productos 
para los que existe demanda insatisfecha.

Un factor adicional, que posibilita la reproducción de esta situación a lo largo del tiempo se evidencia al considerarse la falta de acciones de carácter colectivo entre los actores que realizan la recolección. Si bien es cierto que la escasez de instancias institucionales-asociativas y la carencia de experiencias de cooperación o asociativismo son características comunes en diversos sectores productivos en la región (véase, por ejemplo respecto a la cerveza artesanal de Bariloche, Colino et al., 2017), quienes resultan más perjudicados de esta falta de coordinación en la CVRM son los actores más vulnerables, socialmente hablando. En los últimos años se han realizados esfuerzos tendientes a establecer una cooperativa de recolectores de Rosa Mosqueta, pero la misma no prosperó en parte por una aducida falta tiempo de los mismos recolectores, lo cual se considera un indicador de la falta de percepción de la utilidad que podrían encontrar en este tipo de asociatividad (Entrevista a empresario del sector, 2016). Por lo mencionado en este apartado, en el cual se ha mostrado la dependencia de los recolectores respecto de los acopiadores, se puede afirmar que en el vínculo de estos dos conjuntos de actores existe una especie de governance de tipo "cautiva", según la categorización de Gereffi et al. (2005).

Cabe resaltar el hecho de que, en el marco del trabajo del Programa PAR de la Universidad Nacional de Río Negro (UNRN) y el Ministerio de Ciencia, Tecnología e Innovación Productiva (MINCYT), en conjunto con el INTI Delegación Bariloche, se indagó sobre la posibilidad de generar tecnologías de carácter social ${ }^{6}$ que, como resultado esperado de su desarrollo e implementación, permitan incrementos en la productividad de la cosecha y en la seguridad personal de los recolectores. Asimismo, se espera que estos desarrollos tecnológicos puedan fungir de factores catalizadores del asociativismo de los recolectores. Sin embargo, hasta la fecha estos esfuerzos han sido infructuosos.

\subsection{La territorialidad (o alcance geográfico) de la CVRM}

En términos generales, puede afirmase que la cadena de valor de la Rosa Mosqueta tiene una fuerte raigambre local. Esto implica que no existan grandes costos asociados a logística, al menos hasta su comercialización. La excepción a esta afirmación es la elaboración de productos cosméticos, los cuales son elaborados en laboratorios habilitados de la ciudad de Buenos Aires, a los que se les envía el aceite, y que luego remiten los productos elaborados a las empresas locales.

Además de la venta por canales minoristas, en los últimos años la producción de Rosa Mosqueta y productos derivados de bajo valor agregado como cascarilla, semillas y aceites esenciales, producidos en Bariloche y zonas aledañas, se venden a empresas de Chile (uno de los principales productores a nivel mundial), aunque no ha sido posible establecer volúmenes ni precios. Cabe mencionar que, a nivel mundial, el comercio de productos silvestres como bayas, frutos, hongos y hierbas, se realiza para su venta a la industria cosmética, farmacéutica, alimenticia (y de aditivos alimenticios) y de fibras. Sin embargo, las estadísticas respecto a su comercio mundial son muy escasas, en parte puesto que los productos recolectados

6 Puntualmente se trata del desarrollo prototipos de una herramienta manual de cosecha específica y de una cosechadora mecanizada. Véase PDTT, 2016a y 2016b. 
en silvestría suelen tener más de un nombre genérico a los fines estadísticos ${ }^{7}$. Respecto a lo Rosa Mosqueta en particular, la dificultad inherente a establecer cantidades de producción y comercio internacional radica en el hecho de que la posición arancelaria en la que se sitúa, (12-1211), es compartida "con cientos de otras especies" vegetales (International Trade Centre, 2010, p. 10).

Tabla 2. Producción mundial de Rosa Mosqueta. 2005. En toneladas.

\begin{tabular}{lc} 
País & Toneladas \\
\hline Chile & 3.806 \\
Rumania & 3.051 \\
Argentina & 1.400 \\
Lesoto & 1.000 \\
Bulgaria & 120 \\
Albania & 50 \\
Macedonia & 10 \\
Serbia y Montenegro & 5 \\
\hline Total Estimado & $\mathbf{9 . 4 4 2}$
\end{tabular}

Fuente: elaboración propia en base a INTA - SIPAN (2005) y International Trade Centre (2007).

Pese a esta dificultad, y según estimaciones propias basadas en las estadísticas disponibles, durante el año 2005 la recolección y cosecha de Rosa Mosqueta habría alcanzado un total de 9.942 toneladas mundiales (INTA - SIPAN, 2005 e International Trade Centre, 2007), siendo los principales productores y su respectiva contribución la que se detalla en la Tabla 2, y representando la quinta producción silvestre del mundo $^{8}$ (International Trade Centre, 2007).

\subsection{El contexto institucional}

En términos generales puede afirmase que la cadena de valor de la Rosa Mosqueta está regulada en Argentina por las normativas vigentes que rigen la producción de alimentos y productos cosméticos que dependen a nivel nacional de la ANMAT. Adicionalmente, a nivel local los municipios ejercen actividades de contralor en cuanto a habilitaciones comercial y bromatológica, esta última vinculada a la elaboración de alimentos. En el caso de la Municipalidad de San Carlos de Bariloche (MSCB), la habilitación municipal está a cargo del área de Inspección General. La MSCB otorga, además, una habilitación bromatológica a los establecimientos de elaboración, y la habilitación comercial necesaria tanto en el caso de establecimientos elaboradores como comercializadores. Cabe resaltar que la ordenanza para la habilitación ha quedado desactualizada, puesto que data de la década del setenta.

Al analizar el contexto institucional en el que se inserta la CVRM se destaca la

7 De hecho, se los suele referir de diversas maneras, tales como: wild collected products, wild crafted products, wild harvested products, wild grown products, biodiverstiy products, non-timber forest products (NTFPs) o non-wood forest products (NWFPs). Esta dispersión terminológica dificulta la realización de estadísticas mundiales (International Trade Centre, 2007).

$8 \mathrm{Si}$ se agregan los datos de INTA para argentina en 2005, la rosa mosqueta quedaría en cuarto puesto por delante Ligonberry o Arándano Rojo (Vaccinium vitis-cáceas), una planta nativa de Eurasia y Norteamérica. 
existencia de instituciones formales (marcos legales y normativos) e informales (convenciones, valores, costumbres) de impacto positivo y otras que restringen el desarrollo del sector. Por un lado, existe un limitado interés en el sector por parte de diversos actores del sector gubernamental como también científico-tecnológico. Pese a ello, algunas instituciones han demostrado una mayor acción respecto a esta cadena de valor. Entre ellas se cuenta el INTA, el INTI, y algunas Universidades Nacionales (UUNN) como la Universidad Nacional del Comahue (UNCo), la Universidad Nacional del Sur (UNS) y la ya mencionada UNRN. Respecto al INTI, cabe mencionar que funcionarios de este instituto han trabajado junto a diversos actores pertenecientes a diferentes eslabonamientos productivos, manteniendo vínculos y proveyendo servicios tecnológicos al sector desde hace algunos años. En tanto que el aporte de la UNS ha girado en torno al estudio de metodologías de optimización del secado de frutos, la UNRN, por medio del programa PAR, ha realizado relevamientos de demandas de innovación vinculadas a los eslabonamientos de recolección y procesamiento de los mismos.

También existe un proyecto de ley nacional que tiene por fin crear "en el ámbito del Ministerio de Agricultura, Ganadería y Pesca de la Nación el Programa de Desarrollo Agroindustrial y Comercial de la Rosa Mosqueta, Programa PROROMO”. El mismo, presentado al Senado de la Nación en 2017 (Expediente 1353/17), tiene aspectos que podrían ser mejorados como por ejemplo el énfasis puesto en "mejorar posición comercial en la exportación" (Art. 3, inc. 9), sin ocuparse de otros eslabones de la cadena de valor. Otro aspecto que podría mejorarse es la incorporación explícita de instrumentos de promoción del desarrollo tecnológico nacional de soluciones específicas, puesto que el proyecto actual plantea "beneficios en la compra de tecnología para mejora de procesos y profesionalización de la actividad" (Art 3. inc. $8)$, sin especificar su origen. Finalmente, sería menester impulsar el tratamiento parlamentario de este proyecto, puesto que su aprobación implicaría un fuerte impulso a esta cadena de valor.

\section{Conclusiones}

En este trabajo se ha realizado una revisión de la cadena de valor de la Rosa Mosqueta en la ciudad de Bariloche y zonas de influencia. A tal fin, se utilizó el enfoque de cadenas globales de valor adaptado con el propósito de poder analizar este entramado productivo teniendo en cuenta, además de los procesos productivos en sí mismos, el alcance geográfico, la estructura de poder y gobernanza y el contexto institucional.

Inicialmente se ha mostrado que la Rosa Mosqueta es una planta que en la zona de Bariloche y zonas aledañas es percibida en forma ambivalente por diversos sectores de la población. A partir de la información provista, se puede concluir que todos los subproductos de la Rosa Mosqueta, sus productos derivados con poco valor agregado (como cascarilla, semillas y aceites), así como también aquellos productos de mayor valor agregado (que constituyen insumos para las industrias alimenticias y cosmética) tienen un potencial de ventas mucho mayor del que actualmente se realiza. Pese a ello se ha mostrado que el principal factor limitante para incrementar el impacto socioeconómico de esta cadena de valor en la actualidad está vinculado 
a la recolección, actividad que tiene la característica de un alto grado de informalidad y de ser "cautiva" de los acopiadores.

A raíz de esa situación, si bien a la fecha se han realizado relevamientos que permitieron identificar posibles herramientas basadas en tecnologías sociales cuya utilización incrementaría la productividad y seguridad de los/as trabajadores/as en la recolección de frutos, aún no se han realizado desarrollos concretos. Esta situación se considera que se vincula, al menos parcialmente, a la falta de organización que los actores sociales que realizan la recolección han mostrado hasta el momento, puesto que son quienes deberían impulsar el desarrollo de estas soluciones tecnológicas. Sin embargo, por su atomización y la falta de coordinación entre ellos, este grupo no cuenta con los medios económicos ni institucionales necesarios para financiar el desarrollo de prototipos de estas soluciones, por más que instituciones como INTI y las UU.NN han propuesto colaboración al respecto.

Otros posibles desarrollos tecnológicos también han sido identificados. Entre estos, uno se orienta a la optimización de la labor de secado de frutos (basados en investigaciones tecnológicas, de la en cual la empresa consultada no tenían conocimiento), y al procesamiento in-situ de la materia prima como estrategia para empoderar a actores sociales de ámbitos periurbanos y, particularmente, rurales.

Asimismo, respecto a la estructura de coordinación y poder, se nota una sustancial asimetría entre recolectores y empresas acopiadoras generándose un estilo de governance donde los primeros son cautivos de las segundas. Esta situación, tiende a dificultar la existencia de incentivos para el incremento de la cosecha anual, al tiempo que constituye una fuente de incertidumbre por parte de los establecimientos que elaboran productos derivados, respecto al aprovisionamiento de materias primas. Una posible solución a este conflicto radicaría en la posibilidad de prestar apoyo a los actores sociales que trabajan en tareas de recolección a fin de que, por medio del establecimiento de asociaciones o cooperativas puedan, además de mejorar el desempeño de su actividad y participar en el desarrollo de tecnologías sociales de cosecha, incrementar su poder de negociación con los acopiadores, quienes en gran medida fijan el precio local.

En cuanto al alcance geográfico de esta cadena de valor, se ha mostrado que la recolección, acondicionamiento y procesamiento se hace principalmente en Bariloche y zonas aledañas. Sin embargo, en lo referido a la elaboración de productos cosméticos, la misma presenta una total dependencia respecto a la ciudad de Buenos Aires constituyendo un área de oportunidad (a fin de bajar costos logísticos) el establecimiento de laboratorios habilitados por ANMAT en la región. Asimismo, si bien es difícil la obtención de estadísticas de carácter mundial, es posible estimar que la Argentina ocupa un lugar relevante a nivel global en cuanto al volumen recolectado de este fruto, pese a que, a diferencia de lo que ocurre en Chile (el principal productor) no se realizan explotaciones basadas en el cultivo sistematizado.

Finalmente en cuanto al contexto institucional, cabe mencionarse que con excepción del INTI y, algunas UUNN el apoyo de instituciones del sistema científico tecnológico ha sido limitado y no se ha enfocado suficientemente en el aprovechamiento 
comercial de esta especie vegetal. Por ello se espera que este artículo, que deberá ser profundizado en futuros trabajos, pueda constituir un insumo para el desarrollo de políticas públicas tendientes a dinamizar el sector y, con él, la región.

\section{Referencias bibliográficas}

Barros Biscari, R. M. (2010). Los saberes colectivos locales como factores del anclaje territorial. EI SIAL de la Rosa Mosqueta rubiginosa de la Patagonia Argentina. 116TH EAAE Seminar "Spatial Dynamics in Agrifood Systems: Implications for Sustainability and Consumer Welfare", 1-9. Parma, Italia.

Cameroni, G. (2013). Ficha Técnica de Rosa Mosqueta. Buenos Aires, Argentina: Subsecretaría de Agregado de Valor y Nuevas Tecnologías.

Cattan Lavin, M. ( 2007). Equipamiento para la Recolección de Rosa Silvestres (tesina de grado). Universidad de Chile, Santiago de Chile, Chile.

Civitaresi, H.; Niembro, A. y Sarmiento, J. (2015). Procesos de construcción, fortalecimiento y expansión de complejos agroalimentarios y su impacto sobre el desarrollo territorial: una aproximación a enfoques analíticos y metodológicos. IX Jornadas Interdisciplinarias de Estudios Agrarios y Agroindustriales Argentinos y Latinoamericanos. Universidad de Buenos Aires, Buenos Aires, Argentina.

Colino, E., Civitaresi, H. M., Capuano, A., Quiroga, J. M., y Winkelman, B. (2017). Análisis de la estructura y dinámica del complejo cervecero artesanal de Bariloche, Argentina. Pilquen Sección Ciencias Sociales, 20(2), 79-91.

Damascos, M. A. (2008). La rosa mosqueta y otras rosas. San Carlos de Bariloche, Argentina: UNComahue.

Espinoza, T., Valencia, E., Quevedo, R. y Díaz, O. (2016). Importancia y propiedades físico química de la Rosa mosqueta (R. canina, R. rubiginosa): una revisión. Scientia Agropecuaria, 7(1), 67-78.

Gereffi, G. (1994) The organization of buyer-driven global commodity chains: How U.S. retailers shape overseas production networks, en Gereffi G. y Korzeniewicz M. (Eds.), Commodity chains and global capitalism. Westport, Irlanda: Praeger.

Gereffi, G. (1995). Global production systems and Third World development. En B. Stallings (Ed.), Global change, regional response: The new international context of development. Cambridge, Inglaterra: Cambridge University Press.

Gereffi, G. (1999). International trade and industrial upgrading in the apparel commodity chain. Journal of International Economics. 48(1), 37-70.

Gereffi, G., Humphrey, J. y Kaplinsky, R. (2001). Introduction: Globalization, value chains and development. IDS bulletin, 32(3), 1-8. 
Gereffi, G., Humphrey, J. y Sturgeon, T. (2005). The governance of global value chains. Review of International Political Economy, 12(1), 78-104.

INTA - SIPAN. (2005). Sistema de soporte de decisiones para la producción agrícola de los valles cordilleranos patagónicos. Recuperado de: http://sipan.inta.gov. ar/productos/ssd/vc/

INTA AER El Bolsón. (2013). Control mecánico de la Rosa Mosqueta en un establecimiento ganadero del paraje Mallín Ahogado, El Bolsón, Argentina: Ediciones INTA.

International Trade Centre. (2007). Overview of World Production and Marketing of Organic Wild Collected Products. Ginebra, Suiza: UNCTAD/WTO.

International Trade Centre. (2010). MNS Medicinal Plant and Extracts. Ginebra, Suiza : UNCTAD/WTO.

Kaplinsky, R. y M. Morris (2000). A Handbook for Value Chain Research. Prepared for the IDRC.

Lloyd, C. E. (2014). Avances en el control de Rosa Mosqueta. Esquel, Argentina: INTA.

Madariaga, M. (2007). La agroindustria en Bariloche: principales características. Presencia, 17(51), 30-35.

Ohaco, E., Mabellini, A., y Márquez, C. (2011). Pretratamientos para acelerar el secado de frutos de rosa mosqueta. Presencia, 22(56), 34-37.

Patel, S. (2013). Rose hips as complementary and alternative medicine: overview of the present status and prospects. Mediterranean Journal of Nutrition and Metabolism, 6(2), 89-97.

PDTT. (2016a). Demanda de Innovación: Diseño y desarrollo nacional de herramientas manuales para cosecha de Rosa Mosqueta. Recuperado de: http://www. Innovaciónargentina.gob.ar/? Itemid=125

PDTT. (2016b). Demanda de Innovación: Cosechadora mecanizada y portatil de Rosa Mosqueta. Recuperado de: http://www. Innovaciónargentina.gob. ar/? Itemid=125

PDTT. (2016c). Demanda de Innovación: Planta móvil de procesamiento de Rosa Mosqueta. Recuperado de: http://www. Innovaciónargentina.gob. ar/? Itemid=125

PDTT. (2017). Dispositivo perforador de pseudofrutos de Rosa Mosqueta. Recuperado de: http://www. Innovaciónargentina.gob.ar/?ttemid=125

Porter, M. (1980). Competitive Strategy: Techniques for Analyzing Industries and Competitors. Nueva York, EE.UU.: The Free Press. 
Porter, M. (1985). Competitive Advantage: Creating and Sustaining Superior Performance. Nueva York, EE.UU.: The Free Press.

\section{Entrevista}

Quiroga, J. M. (Entrevistador). (06/05/2016). Entrevista a empresario del sector. 